\title{
ECKART BIRNSTIEL
}

\section{ASYL UND INTEGRATION DER HUGENOTTEN IN BRANDENBURG-PREUSSEN}

Im April 2006 führte ein Meinungsforschungsinstitut im Auftrag des "Spiegel $\lll$ in Deutschland eine Umfrage durch, die unter dem provokanten Titel

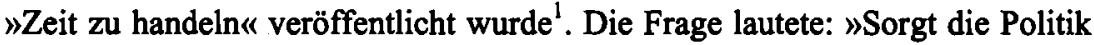
ausreichend für die Integration ausländischer Mitbürger?«. 33 Prozent der rund tausend Befragten antworteten mit »ja« und 60 Prozent mit »nein«; der Rest konnte oder wollte sich hierzu nicht äußern. Ich selbst hätte mich wahrscheinlich auch einer Antwort enthalten, denn die Frage ist tendenziös. Sie setzt bereits voraus, daß Integration durch politische Maßnahmen herbeigeführt werden kann, und läßt offen, was in diesem Zusammenhang als wausreichend« verstanden wird. Sie hätte besser lauten sollen: »Kann die Politik für die Integration ausländischer Mitbürger sorgen?«.

Genau diese Frage wird sinngemä $B$ im Mittelpunkt der nachfolgenden Überlegungen zur Geschichte der gegen Ende des 17. Jahrhunderts aus Frankreich nach Brandenburg-Preußen eingewanderten hugenottischen Glaubensflüchtlinge stehen, wobei freilich auch andere Probleme in den Gesichtskreis treten: War ihre Integration im Hohenzollernstaat überhaupt politisch erwünscht? In welchem Maße wurde ihre Integration von der Fremdwahrnehmung seitens ihrer deutschen Umwelt mitbestimmt? Wie kann man den Grad der Integration einer Immigrantengruppe messen? In welchen Phasen und Zeiträumen verlief der Integrationsprozeß der Hugenotten? Und schließlich: ist das historische Beispiel der Hugenotten mit der aktuellen Lage diasporischer Gruppen vergleichbar?

Der Begriff "Integration« selbst bedarf jedoch vorab einer Klarstellung. Wenn wir davon ausgehen, daß jede Gesellschaft ihre eigene Ordnung hat, die den politischen und ökonomischen Bedingungsrahmen des sozialen Zusammenlebens aller ihrer Glieder definiert und die den Respekt eines einheitlichen ethischen Wertesystems voraussetzt, dann bedeutet Integration für eine Gruppe von Einwanderern, die allgemeinen Verhaltensregeln der Aufnahmegesellschaft zu übernehmen und diese auch im Innenverhältnis der eigenen Gruppe lebensweltlich umzusetzen, was schließlich zu deren "Ent-Fremdung" und kultureller Auflösung führt.

${ }^{1}$ Der Spiegel 17/2006, S. 20. 
Paradoxerweise ist Integration also erst dann erreicht, wenn niemand mehr über sie spricht.

\section{Zur identitären Rekonstruktion der Hugenotten im Refuge}

Eine Flüchtlingsidentität erlangt man erst auf der Flucht. Bevor der Flüchtling zum Einwanderer wird, hat er bereits eine Zeit der Auswanderung und der Wanderung auf den Straßen des Exils hinter sich. Je weiter er sich dabei von seiner Heimat entfernt, desto fremder erscheint ihm seine Umwelt, und desto fremder erscheint er auch den Menschen, denen er auf seinen Wegen begegnet. Aus dem Auswanderer wird zunächst einmal ein Fremder. Seine Suche nach einer neuen Heimat ist auch eine Suche nach neuen gesellschaftlichen Einbindungen, letztlich eine Suche nach positiver Selbstbestätigung, nach einer neuen, lebenswerten Identität. Das gilt für Flüchtlinge, die alles hinter sich gelassen haben, um ihren Glauben nicht verleugnen zu müssen, in besonderem Maße: für sie gibt es kein Zurück mehr; sie sind auf Gedeih und Verderb darauf angewiesen, sich in der Fremde neu zu erfinden.

In unserem durch internationalen Massentourismus und globale Informationsnetze geprägten Zeitalter können wir es uns kaum noch vorstellen, was Fremdheit für die Menschen vergangener Jahrhunderte bedeutete. Für sie begann das Elend ${ }^{2}$ bereits jenseits der Hügel, wo die Leute mit einem anderen Zungenschlag redeten, den Gürtel anders banden und mit anderen Maßen und Gewichten hantierten. Heimat war dort, wo man alles ohne weitere Erklärung verstand: Sobald man stutzte, wußte man, daß man nicht mehr zuhause war.

Das galt auch noch für das Frankreich Ludwigs XIV. Frankreich war zu jener Zeit zwar ein zentralistisch regierter Staat mit leistungsfähigen politischen Verwaltungsstrukturen, hatte es jedoch noch nicht zu nationaler oder auch nur kultureller Einheit gebracht. Das Frankreich, aus dem die Hugenotten kamen, war ein territorialstaatliches Gebilde mit einer Unzahl von Regionalkulturen und -sprachen, Sitten und Gebräuchen, Weltanschauungen und Lebensweisen. In Frankreich lebten zu jener Zeit noch keine „Franzosen«, sondern Wallonen, Normannen, Bretonen, Gascogner, Basken, Provenzalen und eine Vielzahl weiterer Völker, deren einzige Gemeinsamkeit darin bestand, daß sie der selben politischen Obrigkeit unterworfen waren.

Auf dem Weg ins Refuge trafen also Menschen aufeinander, deren Lebenswelten sich zuvor in Frankreich kaum berührt hatten und die daher auch kein verbindendes Gruppenbewußtsein hatten entwickeln können. Sie mußten sich

2 „Elend" war im historischen deutschen Sprachgebrauch gleichbedeutend mit »Fremde« und »Exil«. 
ihre kollektive Identität erst in der Fremde erarbeiten. Hierbei kam ihnen vor allem zugute, daß sie über eine gemeinsame Sprachebene verfügten, die sich aus ihrer religiösen Glaubenskultur ergab. Dieser Punkt ist weniger banal, als es auf den ersten Blick erscheinen mag, denn auch die französischen Reformierten hatten in ihrer Heimat im Alltag meist kein Französisch, sondern ihre lokalen Idiome gesprochen; man denke nur an die in der - überwiegend protestantischen - Südhälfte Frankreichs verbreitete okzitanische Sprache mit ihrer Vielzahl regionaler Mundarten ${ }^{3}$. Neben ihren Heimatdialekten waren die Refugiés jedoch auch mit der französischen Sprache vertraut, die über die Bibel ihren Eingang in den Kulturkreis der reformierten Protestanten gefunden hatte. Der Gott Calvins sprach zu seinen Hugenotten französisch, und diese lasen täglich in der Schrift, hörten die Predigt auf französisch, beteten auf französisch und sangen ihre Psalmen auf französisch ${ }^{4}$. Dieses seit den Zeiten der Reformation bestehende Nebeneinander von französischer lingua sacra und dialektaler lingua franca hatte im Laufe der Generationen zu einer strukturellen Zweisprachigkeit geführt, die es einem Hugenotten aus dem Süden des Landes erlaubte, sich mit einem Glaubensgenossen aus dem Norden einigermaßen verständlich zu unterhalten, während die sprachliche Kommunikation mit dem katholischen Nachbarn natürlich weiterhin im ortsüblichen patois erfolgte.

Im europäischen Refuge, diesem bunten Sammelbecken von Flüchtlingen aus allen französischen Landstrichen und Partikularkulturen, waren die muttersprachlichen Regionalidiome der Refugiés zu deren allgemeiner Verständigung untauglich und wurden schnell von der französischen Sprache verdrängt, die von den Hugenotten als alleinige Verkehrssprache angenommen wurde. Bevor sie sich den einheimischen Sprachen ihrer Aufnahmeländer öffneten und damit wiederum zweisprachig wurden, erarbeiteten sie sich also erst einmal eine gemeinsame französische Sprachebene: eine Kulturleistung ersten Ranges, die dazu führen sollte, daß nicht etwa die in Frankreich lebenden Franzosen, die erst wesentlich später zu sprachlicher Einheit gelangten, zu den Pionieren der Frankophonie wurden, sondern vielmehr die aus Frankreich geflohenen Protestanten ${ }^{5}$. Es war die hugenottische Diaspora, die die französische Sprache in aller Herren Länder trug.

Die »Französierung « der Hugenotten im Refuge wurde dort auch noch von einem weiteren Element begünstigt: von der positiven Fremdwahrnehmung ihrer Gruppe durch die einheimischen Gesellschaften. Für diese waren die Hugenotten schlicht Franzosen und wurden als Repräsentanten der französischen Hochkultur begriffen, welche in den Versen eines Corneille und eines Racine sowie in der Prachtarchitektur der Epoche Ludwigs XIV., in der Pari-

\footnotetext{
${ }^{3} \mathrm{Vgl}$. Marcel COHEN, Histoire d'une langue: le français (des lointaines origines à nos jours), Paris 1987, S. 78-82.

${ }^{4}$ Vgl. Janine GARRISSON, L'Homme protestant, Brüssel 1986 (Historiques, 33), S. 78-81.

${ }^{5}$ Vgl. COHEN, Histoire d'une langue (wie Anm. 3), S. $181 \mathrm{f}$.
} 
ser Mode und in den feinen Manieren, im hochwertigen Handwerk und in der Garten- und Küchenkunst ihren europaweit bewunderten Ausdruck gefunden hatte. Diese spontane Gleichsetzung von "Hugenotten« und »Franzosen « war freilich ein Mißverständnis, da die Hugenotten nicht zu den Protagonisten der höfischen Kultur von Versailles zählten, sondern vielmehr zu den Opfern der absolutistischen Machtentfaltung des Sonnenkönigs und der Glaubensdiktatur der Gallikanischen Kirche. Doch dieses Mißverständnis sollte sich für sie als segensreich erweisen. Die Hugenotten genossen im Refuge von Anfang an den Status einer hochgeschätzten Minderheit, deren Zuwanderung von den einheimischen Bevölkerungen als ein zivilisatorischer Gewinn empfunden wurde. Sie öffneten den anderen Nationen ein Fenster in den bewunderten Garten Frankreich ${ }^{6}$.

Die Hugenotten kamen also nicht als fest strukturierte Gruppe in ihre Aufnahmeländer. Sie entwickelten sich erst auf den Wegen des Exils zu einer solchen. Im Zuge ihrer identitären Rekonstruktion entstand ein Phänotypus des französischen Protestanten, wie er sich zuvor in Frankreich gar nicht hatte herausbilden können. Er vereinigte in sich religiöse Unbeugsamkeit, die Erfahrung von Flucht und Fremdheit, einen mit ihrer europaweiten Verbreitung einhergehenden Kosmopolitismus und das Bekenntnis zu kultureller Einheit. Fortan bildeten sie eine eigene französische Nation in der Diaspora.

\section{Zur Aufnahme der Hugenotten in den Hohenzollernstaat}

Der brandenburgische Kurfürst Friedrich Wilhelm betrieb großen Aufwand, um die auf den Wegen des Exils herumirrenden Hugenotten in seine Länder zu ziehen: Seine Flüchtlingskommissare in Amsterdam, in Frankfurt am Main und in Köln waren angewiesen, die Refugiés zunächst auf Staatskosten nach Hamburg oder in das hohenzollernsche Herzogtum Kleve geleiten zu lassen, von wo aus sie auf geeignete Siedlungsorte verteilt werden sollten. Sicherlich spielten bei dieser massenhaften Anwerbung hugenottischer Exulanten wirtschaftliche Gründe eine wichtige Rolle, aber der Kurfürst ließ sich mindestens ebenso stark von religiösen Motiven leiten. Wenn dieser in der Präambel des Potsdamer Edikts (29. Oktober 1685) von seinem gerechten Mitleiden, welches Wir mit solchen Unsern, wegen des heiligen Evangelii und dessen reiner

\footnotetext{
${ }^{6}$ Für Brandenburg bringt Rudolf von THADDEN dieses Zivilisationsgefälle auf die treffende Formel: „Nicht also Dritte Welt am Kurfürstendamm, sondern Erste Welt in den Ackerbürgerstädten Brandenburgs. Nicht Türken in Berlin, sondern Berliner in der Türkei«; Rudolf von THADDEN, Einwanderer in fremdem Land. Die Hugenotten in der ständischen Gesellschaft Brandenburg-Preußens, in: DERS., Nicht Vaterland, nicht Fremde. Essays zu Geschichte und Gegenwart, München 1989, S. 9-18, hier S. 9.
} 
Lehre angefochtenen und bedrengten Glaubens $=$ Genossen billig haben müssen $^{7}$ spricht, so sollte ihm hier keine Hypokrisie unterstellt werden. Wäre es ihm ausschließlich um eine »Repeuplierung« seiner während des Dreißigjährigen Krieges entvölkerten Territorien und um einen Anschub der seitdem regressiven brandenburgischen Nationalökonomie gegangen, so hätte er bestimmt den zeitgleich mit den ersten Hugenotten ${ }^{8}$ ins Land gekommenen österreichischen Juden, die sich hierzu ebenso anboten, in seinem sie betreffenden Niederlassungsedikt (20. Mai 1671) die selben Gerechtigkeiten, Freyheiten und Praerogativen zugestanden, mit denen er die Ansiedlung der $\mathrm{Hu}-$ genotten zu befördern gedachte. $\mathrm{Daß}$ der Große Kurfürst zu diesem Schritt nicht bereit war ${ }^{9}$, läßt klar erkennen, daß sich demographische, ökonomische und ethisch-religiöse Motive in seiner die Hugenotten betreffenden Aufnahmepolitik durchaus die Waage hielten ${ }^{10}$.

Erstaunlicherweise findet sich im Potsdamer Edikt keinerlei Hinweis auf den völkerrechtlichen Status der hugenottischen Zuwanderer: Weder wird ihnen die französische Staatszugehörigkeit im Zuge einer kollektiven Denaturalisation aberkannt, noch wird ihnen abverlangt, auf den politischen Souverän ihres Aufnahmelandes den Untertaneneid zu leisten. Es handelte sich bei ihnen, modern gesprochen, um staatenlose Personen, die durch den Akt ihrer illegalen Abwanderung aus Frankreich ihre Qualität als natürliche Untertanen (sujets naturels) der französischen Krone verloren hatten ${ }^{11}$, die aber andererseits noch kein neues politisches Treueverhältnis eingegangen waren. Die hu-

${ }^{7}$ Alle Zitate des Potsdamer Edikts folgen dessen zweisprachiger Wiedergabe in Eduard MURET, Geschichte der Französischen Kolonie in Brandenburg-Preußen, unter besonderer Berücksichtigung der Berliner Gemeinde, Berlin 1885, S. 301-306.

${ }^{8}$ Eine erste Gruppe hugenottischer Glaubensflüchtlinge hatte Brandenburg um 1670 erreicht; vgl. Eckart BIRNSTIEL, Die Hugenotten in Berlin. Eine Gemeinde auf der Suche nach ihrer Kirche, in: Rudolf von THADDEN, Michelle Magdelaine (Hg.), Die Hugenotten, 1685 1985, München 1985, S. $115 f$.

${ }^{9} \mathrm{Vgl}$. die aufschlußreiche Gegenüberstellung beider Edikte in Brigitte SCHEIGER, Juden in Berlin, in: Stefi JERSCH-WENZEL, Barbara JOHN (Hg.), Von Zuwanderern zu Einheimischen. Hugenotten, Juden, Böhmen, Polen in Berlin. [Mit Beiträgen von] Eckart Birnstiel und Andreas Reinke, Brigitte Scheiger, Eva-Maria Graffigna, Gottfried Hartmann, Berlin 1990, S. 153-488, hier S. $167 f$.

${ }^{10}$ Diese Beobachtung wird auch durch den Artikel 13 des Potsdamer Edikts gestützt, in dem Einwanderer römisch-katholischer Konfession aus dem Kreise seiner Begünstigten ausgeschlossen werden.

${ }^{11}$ Kraft der entsprechenden Gesetzestexte der Regierung Ludwigs XIV. waren die Glaubensflüchtlinge aus dem französischen Untertanenverband ausgeschlossen worden; vgl. "Édit interdisant à tous les sujets du roi de s'installer à l'étranger« (5. August 1669); »Déclaration interdisant aux protestants de vendre leurs biens immobiliers ou la totalite de leurs biens meubles, et à tous sujets du roi de se fixer à l'étranger« (14. Juli 1682; bekräftigt am 7. September 1682 und am 31. Mai 1685); katalogisiert in Arie Theodorus van DEURSEN, Professions et métiers interdits. Un aspect de l'histoire de la révocation de l'Édit de Nantes, Groningen 1960, S. 363-365. 
genottischen Zuwanderer waren also im Rechtsverständnis der Zeit »Fremde« (aubains) $^{12}$, denen das Potsdamer Edikt nicht mehr und nicht weniger als eine sichere und freye retraite in alle unsere Lande und Provincien in Aussicht stellte. Anders gesagt: Die Hugenotten wurden 1685 in Brandenburg kraft eines kurfürstlichen Gnadenaktes als Asylanten eingelassen, wobei die Dauer ihres Asyls im übrigen - ebenso wie ihr Status - nicht näher festgelegt wurde ${ }^{13}$.

Dieser Zustand sollte noch einige Zeit anhalten. Zwar verlangte Kurfürst Friedrich III. kurz nach Beginn des von der Großen Allianz gegen Frankreich geführten Pfälzischen Krieges (1688-1697) von den in seinem Militärdienst stehenden Hugenotten die individuelle Ablegung eines Treueeides ${ }^{14}$, doch erklärten sich diese damit nicht rechtskräftig zu dessen Untertanen, sondern beschworen lediglich, sich künftig in allem so zu verhalten comme il convient et appartient à un fidèle sujet ${ }^{15}$, also in der Art, wie es auch einem streuen Untertanen geziemt und zukommt«. Und selbst als alle Bemühungen der Refugiés gescheitert waren, ihre bedingungslose Rückkehr nach Frankreich in den Friedensvertrag von Rijswijk aufnehmen zu lassen ${ }^{16}$, wurden sie in Brandenburg den eingeborenen Untertanen feudalrechtlich noch immer nicht gleichgestellt. Erst das am 13. Mai 1709 von König Friedrich I. erlassene Naturalisationsedikt erlöste sie aus ihrer prekären Lage; es verfügte,

daß alle, in Unseren Landen bereits établirte, und künffig noch sich darin établirende Refugirte, es mögen dieselben aus Franckreich, oder anderweitlich, der Religion halber, vertrieben seyn, nicht anders, als Unsere eingebohrne Unterthanen, so bald sie sich Uns, und Unserm Königlichen Haus, mit Eydes=Pflichten verbindlich gemacht haben werden, consideriret, geachtet und gehalten werden sollen. [...] Wir setzen, ordnen, und wollen demnach, daß [...] alle Refugirte, und ihre Kinder, von was Nation und Stande sie auch seyn, nicht anders,

${ }^{12}$ Dieser Tatbestand wird in Artikel 7 des Potsdamer Edikts direkt angesprochen; die Hugenotten werden dort vom Heimfallrecht auf ihre Verlassenschaft (droit d'aubaine) und anderen dergleichen Beschwerden, womit die Fremde in andern Königreichen, Landen und republiquen belegt $z$ werden pflegen, gäntzlich befreyet.

${ }^{13}$ Das Zugeständnis von fiskalischen Freijahren sowie der in Artikel 5 enthaltene Passus bezüglich der unentgeltlichen Übertragung von Wohnraum für sie, ihre Erben und Erbens $=$ Erben lassen allerdings darauf schließen, daß der Große Kurfürst eine lange Konzessionsdauer unterstellte.

${ }^{14}$ Dieses Verfahren war auch bei der Rekrutierung ausländischer Söldner üblich.

${ }^{15}$ Der Text dieses in französischer Sprache abzulegenden Eides (17. Februar 1690) ist wiedergegeben in Henri TOLLIN, Geschichte der Französischen Colonie von Magdeburg. Jubiläumsschrift, 3 Teile in 6 Bden., Halle/Saale 1886-1894, Bd. III.2, S. 31; Tollin bezeichnet ihn allerdings irrig als »Unterthaneneidk.

${ }^{16}$ Vgl. Frank PUAUX, Essai sur les négociations des réfugiés pour obtenir le rétablissement de la religion réformée au traité de Ryswick (octobre 1697), in: Bulletin historique et littéraire de la Société de l'histoire du protestantisme français (BSHPF) 16 (1867), S. 257-267 [première partie] und S. 305-316 [deuxième partie]; Charles READ, Les démarches des réfugiés huguenots auprès des négociateurs de la paix de Ryswick pour leur rétablissement en France, 1697, in: BSHPF 40 (1891), S. 169-188; DERS., Les Réfugiés huguenots, lors du traité de Ryswick, in: BSHPF 40 (1891), S. 384-387. 
als Unsere angebohrne Unterthanen consideriret, sie auch überall, und, wo es nöthig, von Uns, so wol hier, als in auswärtigen Landen [...], so ihnen von Rechtswegen zukömbt, geschützet werden sollen ${ }^{17}$.

Erst vierundzwanzig Jahre nach Erla $\$$ des Potsdamer Asyledikts wurden die hugenottischen Einwanderer also in ihrer Gesamtheit zu Untertanen der Hohenzollern erklärt. Weshalb ihre Naturalisation in Brandenburg - aber auch in anderen Ländern des Refuge ${ }^{18}$ - erst derart spät erfolgte, ist bisher von der Forschung nicht schlüssig dargelegt worden ${ }^{19}$. Halten wir hier also lediglich fest, daß die Hugenotten im Hoheitsbereich der Hohenzollern bis 1709 als Fremde galten ${ }^{20}$.

Das Rechtsverständnis der Zeit erlaubte keine juristische Gleichstellung der Fremden mit den natürlichen Untertanen eines politischen Souveräns. Ihr Status konnte nur über ein privilegium - ein personen- oder gruppenbezogenes, außerhalb des Landrechts stehendes Sondergesetz - geregelt werden. Wie das bereits erwähnte Beispiel der Juden zeigt, mußte ein derartiges Privileg nicht zwangsläufig eine Vorzugsbehandlung der betreffenden Fremdengruppe vorsehen: Es lag im freien Ermessen des Landesherrn, welchen sozialen und ökonomischen Platz er den Fremden innerhalb seines Staatsvolkes zuwies und auf welche Weise er deren Einordnung in seine politischen, juristischen, fiskalischen und nicht zuletzt auch kirchlichen Regierungs- und Verwaltungsstrukturen vornahm.

Im Falle der Hugenotten entschied sich der Große Kurfürst in seinem Potsdamer Edikt bekanntlich gegen deren Integration in seinen Untertanenverband: Sie sollten nicht individuell in sein Staatsvolk eingegliedert, sondern seinem Staatswesen als geschlossene Gruppe angegliedert - oder zugeordnet - werden. Er schuf damit nicht mehr und nicht weniger als eine neue Körperschaft, die neben den bereits verfaßten Sozialverbänden seines Landes bestehen sollte. Nicht die Integration der Hugenotten war das Ziel seiner Aufnahmepolitik, sondern deren Inkorporation.

\footnotetext{
${ }^{17}$ Zit. nach MURET, Geschichte der Französischen Kolonie (wie Anm. 7), S. 307.

${ }^{18}$ In England wurde den Refugiés zwischen 1709 und 1712 die allgemeine Naturalisierung gewährt; vgl. Susanne LACHENICHT, Migration, Migrationspolitik und Integration. Hugenotten in Brandenburg-Preußen, Irland und Großbritannien, in: Manuela BÖHM, Jens HÄSELER, Robert VIOLET (Hg.), Hugenotten zwischen Migration und Integration. Neue Forschungen zum Refuge in Berlin und Brandenburg, Berlin 2005, S. 37-58, hier S. 42 und S. 46; in den Niederlanden erfolgte ihre kollektive Naturalisierung im Oktober 1715; vgl. Hans BOTS, René BASTIAANSE, Die Hugenotten und die niederländischen Generalstaaten, in: THADDEN, MAGDELAINE, Die Hugenotten (wie Anm. 8), S. 61.

${ }^{19}$ Es handelt sich hier um eines der großen Desiderata der vergleichenden Hugenottenforschung, das hoffentlich bald seinen Bearbeiter findet.

${ }^{20}$ Das Potsdamer Edikt bezeichnet sie als Frantzosen, als Frantzösische Leute, von der Religion, als gedachte Leute von der Religion, als Frantzösische Glaubens=Genossen, meistens aber als Unsere Evangelisch=Reformierten Glaubens=Genossen Frantzösischer Nation.
} 
Folgerichtig erhielten sie ihre eigene - dem Prinzip des Summepiskopats des Landesherrn freilich angepaßte - Kirchendisziplin ${ }^{21}$ sowie ihre eigene - nur in den oberen Instanzenzügen mit der brandenburgischen Justiz verknüpfte - Gerichtsbarkeit ${ }^{22}$ und damit auch ihre eigene Kolonieverwaltung ${ }^{23}$. Auch in ihrem häuslichen und wirtschaftlichen Leben erfuhren sie eine Sonderbehandlung: Es wurde ihnen freier Wohnraum zugewiesen; ihre unternehmerischen Aktivitäten wurden subventioniert; ihre steuerliche Belastung wurde gegenüber den Einheimischen deutlich verringert, und es wurde Sorge getragen, daß die Ackerbauern unter ihnen auf dem Land der kurfürstlichen Domänenämter - und nicht auf demjenigen der feudalen Gutsherrschaften - angesiedelt wurden ${ }^{24}$.

Das Potsdamer Edikt wies den Hugenotten eine parallele Existenzform zu, die bis über die Mitte des 18. Jahrhunderts hinaus neben der einheimischen Gesellschaft bestand.

\section{Zur Integration der Hugenotten in die preußische Gesellschaft}

Die soziokulturelle und schließlich auch politische Auflösung der preußischen Hugenottenkolonie wurde durch vier relativ dicht aufeinanderfolgende, aber voneinander unabhängige Gesetzesakte beschleunigt: die Einführung des Wahlbürgerrechts in Preußen durch Friedrich II. (1772); das Toleranzedikt des französischen Königs Ludwig XVI. (1787); das zugunsten der Glaubensflüchtlinge und ihrer Nachkommen erlassene Reparationsgesetz der Französischen Nationalversammlung (1790), und schließlich die Kabinettsorder Friedrich Wilhelms III. bezüglich der künftigen Verfassung der Französischen Kolonie in Preußen (1809).

Mit seinem 1772 erlassenen Edikt verfügte Friedrich $\Pi$., daß denen in Köngl. Landen sich niederlaßenden Fremden frey stehen soll, unter Welche Colonien und Gerichte sie sich begeben wollen. Alle Neuankömmlinge - also Einwanderer sowie auch alle während der vorangegangenen friderizianischen

${ }^{21}$ Vgl. BIRNSTIEL, Die Hugenotten in Berlin (wie Anm. 8), S. 123-126.

${ }^{22} \mathrm{Vgl}$. Jürgen WILKE, Rechtsstellung und Rechtsprechung der Hugenotten in BrandenburgPreußen (1685-1809), in: ThadDEN, MAGDELAINE, Die Hugenotten (wie Anm. 8), S. 100 114.

${ }^{23}$ Vgl. Jürgen WEITZEL, Landesherrliche Administrationsmaßnahme zur Eingliederung hugenottischer Flüchtlinge, in: Heinz DuCHHARDT $(\mathrm{Hg}$.$) , Der Exodus der Hugenotten. Die$ Aufhebung des Edikts von Nantes 1685 als europäisches Ereignis, Köln, Wien 1985 (Beihefte zum Archiv für Kulturgeschichte, 24), S. 130-135.

${ }^{24}$ Vgl. Klaus VETTER, Die Hugenotten im System der ostelbischen Gutswirtschaft in der Mark Brandenburg, in: DUCHHARDT, Exodus (wie Anm. 23), S. 141-154, hier S. $147-154$. 
Eroberungskriege unter die preußische Herrschaft gefallenen Personen - hatten demnach innerhalb von drei Monaten zu erklären, ob sie sich der Gerichtsbarkeit der deutschen Magistrate oder derjenigen der Französischen Kolonie unterstellen wollten. Durch dieses Wahlbürgerrecht verlor die Französische Justiz ihren exklusiven Vertretungsanspruch der Hugenotten, da künftig auch Polen und Schlesier sowie später vor allem französische Revolutionsemigranten unter ihre Gerichtsbarkeit fielen ${ }^{25}$. Insofern wurde auch die bisherige konfessionelle Identität zwischen der Französischen Kolonie und der FranzösischReformierten Kirche in Preußen aufgehoben, da es sich bei den Neubürgern der Kolonie in der Regel um Katholiken handelte.

Das Wahlbürgerrecht führte zwar zu einem zahlenmäßigen Anstieg der zwischenzeitlich quantitativ merklich abgefallenen Französischen Kolonie, leitete aber gleichermaßen durch die mit ihm einhergehende konfessionelle Koexistenz der Kolonisten eine Lockerung der Kirchenbindung der Hugenotten ein: Hatten zum Beispiel im Jahre 1725 in Berlin noch über 4500 Kolonisten - bei einer Gesamtzahl von knapp 8000 Refugiés jeden Alters und Geschlechts am Abendmahl in der Französisch-Reformierten Kirche teilgenommen (56\%), so waren es 1785 nur noch an die 1700 der insgesamt rund 6700 Kolonisten $(25 \%)^{26}$. Selbstverständlich war die massenhafte Abkehr vom religiösen Leben auch der fortschreitenden Säkularisierung der preußischen Gesellschaft geschuldet, aber daß die Hugenotten nun ihre Kirche offensichtlich nicht mehr als den Mittelpunkt ihrer Kolonie betrachteten, lag sicherlich auch an dem mit der Einführung des Wahlbürgerrechts einhergehenden Verlust der konfessionellen Identität der Kolonie.

Fünfzehn Jahre nach Einführung des Wahlbürgerrechts in Preußen erließ der französische König Ludwig XVI. im November 1787 ein Edikt »betreffend

\footnotetext{
${ }^{25}$ Es existiert meines Wissens keine historische Monographie über das Wahlbürgerrecht; zur ersten Orientienung über seine Auswirkungen auf die preußischen Hugenottenkolonien vgl. Geheimes Preußisches Staatsarchiv Berlin, Rep. 122, Französisches Ministerialarchiv, 6a, Colonie-Sachen, Generalia, 1684-1804, Nr.10, Wahlbürgerrecht, freie Wahl des Gerichts; Nr. 11, Aufnahme von Fremden zu Colonie-Bürgern, 1779; 7.B.I, Frz. Colonie in Berlin, Gerichtsakten, 1686-1809, Nr. 24, Annahme der Bürger bei der frz. Kolonie zu Berlin; Nr. 57, Beschwerde des Laurent wegen Erschwerung der hiesigen Colonie-Bürger-Annahme, 1788-1789; 7.B.II, Frz. Colonie in Berlin, Einwohnersachen, 1686-1805, Nr. 69, Aufnahme frz. Emigrierter unter die frz. Colonie-Jurisdiction zu Berlin, 1795; Nr. 71, Marquis de St. Maixent, Aufnahme unter hiesiger frz. Coloniegerichtsbarkeit; 23, Frz. Colonie Potsdam, 1694-1801, Nr. 22, Aufnahme frz. Emigrierter unter die Gerichtsbarkeit der Kolonie, 17961797.

${ }^{26}$ Vgl. die Graphiken in François DAvID, Refuge huguenot et assimilation: le cas de la Colonie française de Berlin, in: Eckart BIRNSTIEL (Hg.), avec la collaboration de Chrystel BERNAT, La Diaspora des Huguenots. Les réfugiés protestants de France et leur dispersion dans le monde (XVI'-XVII' ${ }^{e}$ siècles), Paris 2001 (Vie des huguenots, 17), S. 75-97, hier S. 9297.
} 
jene, die sich nicht zur katholischen Religion bekennen ${ }^{27}$. Dieses Toleranzedikt beendete die seit einem Jahrhundert andauernde Diskriminierung der französischen Protestanten, die ihre Geburten, Eheschließungen und Todesfälle fortan nicht mehr durch katholische Geistliche registrieren lassen mußten, sondern von königlichen Justizbeamten in für sie eigens reservierte, kirchenunabhängige Zivilstandsregister einschreiben lassen konnten. Des weiteren gewährte Ludwig XVI. allen seinerzeit in Frankreich lebenden oder in der Folge zuwandernden Nichtkatholiken das unbeschränkte örtliche Niederlassungsrecht, die freie Berufsausübung ${ }^{28}$ und den ungehinderten Genuß aller ihrer Titel, Würden, Besitztümer und Anwartschaften. Den französischen Protestanten blieb freilich der öffentliche Kultus untersagt, da der Katholizismus in Frankreich nach wie vor als Staatsreligion galt.

Die Auswirkungen des Toleranzedikts auf die identitäre Verfassung der preußischen Hugenotten lassen sich nicht konkret messen. Man wird aber davon ausgehen können, daß die veränderte Lage in Frankreich zumindest deren weitere Verunsicherung nach sich zog. Denn eigentlich hätten sie nun in das Land ihrer Väter zurückkehren können, da sie dort keine Benachteiligung, geschweige denn Verfolgung mehr zu befürchten hatten. Ihre bisher sorgsam gepflegte Identität als französische Glaubensflüchtlinge ${ }^{29}$ wurde somit ihres wichtigsten Elementes beraubt: der Unmöglichkeit einer Rückkehr nach Frankreich.

Hatten Wahlbürgerrecht und Toleranzedikt bereits einen tiefgreifenden Identitätswandel der Hugenotten eingeleitet, so wurde dieser durch die Gesetzgebung der französischen Nationalversammlung ${ }^{30}$ abgeschlossen. Nachdem diese bereits in der "Erklärung der Menschen- und Bürgerrechte« (26. August 1789) die Gewissens- und Kultfreiheit aller in Frankreich bestehenden Glaubensgemeinschaften verkündet hatte, bestätigte sie am 10. Juli 1790 in einem Dekret die angestammten Besitztitel und Erbrechte der französischen Glaubensflüchtlinge und ihrer Nachkommen bezüglich ihrer seit 1685 vom französischen Staat beschlagnahmten mobilen und immobilen Habe. Das entsprechende Ge-

${ }^{27}$ Édit Du Roi, Concernant ceux qui ne font pas profession de la Religion Catholique, Versailles, November 1787 (ND der Ausgabe Paris, N.H. Nyon, 1788), in: André ENCREVE, Claude LAURIOL (Hg.), Actes des Journées d'étude sur l'édit de 1787 (Paris 9 et 10 octobre 1987), in: BSHPF (wie Anm. 16), 134 (1988), S. 177-472, hier S. 179-186.

${ }^{28}$ Allerdings unter Ausschluß aller amtlichen Funktionen der königlichen, städtischen und grundherrschaftlichen Gerichtsbarkeit sowie des öffentlichen Unterrichts.

${ }_{29}$ Vgl. Viviane ROSEN-PREST, L'historiographie des huguenots en Prusse au temps des Lumières. Entre mémoire, histoire et légende: J.P. Erman et P.C.F. Reclam, Mémoires pour servir à l'histoire des réfugiés françois dans les États du Roi (1782-1799), Paris 2002 (Vie des huguenots, 23).

${ }^{30}$ Vgl. hierzu Eckart BIRNSTIEL, La France en quête de ses enfants perdus. Mythe et réalité du retour au »pays des ancêtres« des huguenots du refuge, de la Réforme à la Révolution, in: Diasporas. Histoire et Sociétés 8 (2006), S. 22-44. 
setz wurde am 15. Dezember 1790 zusammen mit einer wichtigen Erweiterungsklausel erlassen: Alle im Ausland geborenen Personen, unter deren direkten Vorfahren sich ein französischer Glaubensflüchtling männlichen oder weiblichen Geschlechts befand, wurden zu eingeborenen Franzosen (naturels français) erklärt, sobald sie ihren Wohnsitz in Frankreich nahmen und dort den Bürgereid leisteten. Damit war das Tor nach Frankreich für alle rückkehrwilligen Nachkommen der einstigen hugenottischen Refugiés weit geöffnet: Sie konnten sich die Güter ihrer Vorfahren zurückerstatten lassen und die französische Staatsbürgerschaft annehmen, und zwar ohne Rücksicht auf ihre eigenen religiösen - oder atheistischen - Überzeugungen, ihre nationale Herkunft oder ihre ethnische Zugehörigkeit.

Mit diesem Reparationsgesetz besiegelte die Französische Nationalversammlung das Ende des Refuge. Wer von den betroffenen Nachkommen der hugenottischen Glaubensflüchtlinge keinen Gebrauch von dem Angebot der Wiedereingliederung in Frankreich machte, bekundete damit offen seine vollzogene Integration in die Gesellschaft des Landes, das seine Vorfahren einst aufgenommen hatte. Und das war die überwältigende Mehrheit: Während seiner gesamten Geltungsdauer ${ }^{31}$ wurde das Gesetz vom 15. Dezember 1790 von noch nicht einmal 0,1 Prozent seiner potentiellen Nutznießer in Anspruch genommen ${ }^{32}$. Die meisten Remigranten kamen - wohl aufgrund der sprachlichen Verwandtschaft - aus der Westschweiz, aber die zentral geführten französischen Naturalisationsregister verzeichnen auch dreiundzwanzig Rückkehrer aus Deutschland ${ }^{33}$.

Es war also das französische Reparationsgesetz von 1790, das das hugenottische Refuge endgültig seiner historischen Grundlage beraubte. Die Französische Kolonie in Preußen aber bestand zunächst einmal weiter. Sie wurde erst im Zuge der Preußischen Staatsreformen aufgelöst. In seiner Kabinettsorder vom 30. Oktober $1809^{34}$ bestätigte Friedrich Wilhelm III. die »ursprüngliche Verfassung der Kolonie«, bekräftigte jedoch gleichzeitig: "Unverträglich mit der neuen Organisation ist die isolierte Verfassung der Kolonie in sich, beson-

${ }^{31}$ Der Naturalisationsartikel des Gesetzes vom 15. Dezember 1790 wurde in alle folgenden französischen Staatsverfassungen aufgenommen und erst 1946 im Zuge der Verfassungsänderung der IV. Republik abgeschaff.

${ }^{32}$ Wenn man von einer Zahl von 200000 Glaubensflüchtlingen ausgeht, die Frankreich um 1685 verlassen hatten, so betrug die Gesamtzahl ihrer Gruppe am Ende des 18. Jahrhunderts aufgrund des demographischen Wachstums der Zeit weltweit rund 260000 und um die Mitte des 20. Jahrhunderts rund 350000 Personen; von 1790 bis 1946 nahmen 226 Personen oder $0,06 \%$ der letztgenannten Gesamtzahl - unter Berufung auf das Gesetz vom 15. Dezember 1790 die französische Staatsbürgerschaft an; vgl. hierzu die Magisterarbeit meiner Schülerin Estelle AEBERSOLD, Les rémigrés du refuge huguenot. Le retour des descendants des religionnaires fugitifs en France depuis la loi du 9 décembre 1790 jusqu'au Code de la nationalité du 19 octobre 1945, Université de Toulouse $\amalg$ - Le Mirail 2005.

${ }^{33}$ Vgl. ibid., S. 74.

${ }^{34}$ Wiedergegeben in MURET, Geschichte der Französischen Kolonie (wie Anm. 7), S. $310 f$. 
ders die Vereinigung der einzelnen Gemeinden zu einem abgesonderten Ganzen«. Folgerichtig wurden das Französische Koloniedepartement, das Französische Oberdirektorium und das Französische Oberkonsistorium abgeschafft und das Französische Bürgerrecht beseitigt; das Unterrichtswesen der Hugenotten fiel unter staatliche Aufsicht. Beibehalten wurden lediglich die Gemeindeordnung der Französisch-Reformierten Kirche in Preußen sowie die Französischen Schiedsgerichte, die jedoch nur noch Schlichtungsverfahren im Vorfeld der eigentlichen Prozesse durchführen durften.

Diese Restrukturierungsmaßnahmen bedeuteten das Ende der privilegierten Sonderexistenz der Hugenotten in Preußen. Die Kolonisten wurden nun auch verfassungsrechtlich in die deutsche Gesellschaft entlassen, in der sie bereits seit geraumer Zeit heimisch geworden waren.

\section{Einige Überlegungen zu Integrationsprozessen}

Die Hugenotten haben sich unzweifelhaft in die preußische Gesellschaft integriert und nehmen seither ihren festen Platz im Geschichtsbild des Hohenzollernstaates ein $^{35}$. Das ist insofern erstaunlich, als ihre Integration seitens der brandenburg-preußischen Obrigkeit ursprünglich überhaupt nicht beabsichtigt war: Geplant - oder gar gefördert - wurde ihre politische Eingliederung in die deutsche Gesellschaft bis 1809 nicht. Den Hugenotten wurde - wie auch den Waldensern, den Wallonen, den Böhmen, den Salzburgern und den Juden ${ }^{36}$ eine korporative Sonderexistenz zugewiesen. Zwar galten sie seit 1709 als »eingeborene Untertanen« des preußischen Herrscherhauses, aber dieses legte auf ihre kirchliche, juristische und administrative Abgrenzung von der deutschen Bevölkerung weiterhin großen Wert ${ }^{37}$.

Am Beispiel der Hugenotten wird deutlich, daß zwischen politischer und lebensweltlicher Integration einer Minderheit klar unterschieden werden muß. Während erstere nur kollektiv vollzogen werden kann und einzig und allein vom Willen des Gesetzgebers abhängt, folgt letztere ausschließlich persönlichen Motiven und kann daher nur in individuellem Rahmen stattfinden. Politi-

${ }^{35} \mathrm{Zu}$ diesem Punkt vgl. insbesondere die Ausfiuhrungen in THADDEN, Einwanderer in fremdem Land (wie Anm. 6), S. 9-18, hier S. 13-17.

${ }^{36} \mathrm{Vgl}$. Stefi JERSCH-WENZEL, Minderheiten in der preußischen Gesellschaft, in: Otto BÚsCH, Wolfgang NeUGeBaUER (Hg.), Moderne Preußische Geschichte, 1648-1947. Eine Anthologie, Berlin, New York 1981, S. 486-506.

${ }^{37} \mathrm{Zu}$ den Motiven dieser Abgrenzung vgl. Rudolf von THADDEN, Vom Glaubensflüchtling zum preußischen Patrioten, in: THADden, MagdelaINe, Die Hugenotten (wie Anm. 8), S. 186-197. 
sche Integration kann nur verordnet werden. Lebensweltliche Integration kann nur geschehen.

Dabei kann es durchaus vorkommen, daß beide Prozesse asynchron ablaufen, wie es das Beispiel der Hugenotten ebenfalls zeigt: Als die Französische Kolonie 1809 verfassungsrechtlich aufgelöst wurde, war sie schon seit geraumer Zeit nur noch eine leere institutionelle Hülle. Die Hugenotten hatten inzwischen eine "Abstimmung mit den Füßen « vorgenommen und waren bereits größtenteils in der deutschen Gesellschaft verschwunden ${ }^{38}$. Ihr lebensweltlicher Integrationsproze $B$ verlief unabhängig von staatlichen Vorgaben. Er stand im Widerspruch zu ihnen und ging ihnen historisch gesehen voraus. Die Hugenotten integrierten sich also aus eigener Kraft, wobei ihnen ihre positive Wahrnehmung als »Franzosen« seitens der deutschen Bevölkerung entgegenkam.

Zum besseren Verständnis dieser von der Fremdwahrnehmung einer Gruppe in Gang gesetzten Integrationsmechanismen sei hier auf ein aktuelles Beispiel aus Frankreich verwiesen. Während sich die ersten Immigranten aus Nordafrika noch bewußt als Marokkaner, Algerier und Tunesier begriffen und auf die Aufrechterhaltung ihrer unterschiedlichen kulturellen Prägungen großen Wert legten, wurden sie von der französischen Bevölkerung allgemein als »Maghrebiner« wahrgenommen. Diese externe Qualifikation ist bereits von den in Frankreich geborenen »Kindern der Immigration« willig adoptiert worden, was zur Herausbildung einer »maghrebinischen Nation« geführt hat, die es als solche in Nordafrika überhaupt nicht gibt. Die französischen Maghrebiner verfügen über ihre eigenen kulturellen Zeichensysteme, über ein ausgeprägtes »arabisches« Solidaritätsgeflecht und ein starkes religiöses Zusammengehörigkeitsgefühl. Die »Maghrebinisierung« der Immigranten aus den verschiedenen nordafrikanischen Staaten folgte demnach demselben Grundmuster wie seinerzeit die »Französierung « der Hugenotten. Bis zu diesem Punkt haben die whugenottischen Franzosen« und die »französischen Maghrebiner « - beide in ihrer Zeit - also einen ähnlichen Weg zurückgelegt, woraus geschlossen werden kann, daß ein Leben in der Diaspora grundsätzlich eine identitäre Rekonstruktion der betroffenen Gruppe erzwingt ${ }^{39}$.

Der Vergleich zeigt aber noch etwas anderes. Während die Hugenotten auf die Wertschätzung der Einheimischen trafen und sich daher problemlos integrieren konnten, werden die Maghrebiner von ihrer franko-französischen Umwelt eher abgelehnt und finden daher nur schwer Eingang in die Mehrheitsgesellschaft, was zu gewissen - auch räumlichen - Abkapselungen beider Lebenswelten führt, obwohl seitens der Regierung - anders als zur Zeit der

\footnotetext{
${ }^{38}$ Hierfür spricht auch das Ausbleiben massenhafter Proteste der Hugenotten gegen die Auflösung ihrer Kolonie; diese Thematik bedarf jedoch noch einer näheren Untersuchung.

${ }^{39} \mathrm{Vgl}$. hierzu insbesondere Chantal BORDES-BENAYOUN, Dominique SCHNAPPER, Diasporas et Nations, Paris 2006.
} 
Hugenotten - eine aktive Integrationspolitik der Bewohner der periurbanen cités betrieben wird ${ }^{40}$. Auch die Aufnahmebereitschaft der Einheimischen läßt sich also nicht staatlich verordnen. Sie folgt ihrer eigenen emotionalen Logik, wobei vermutlich auch der religiöse Faktor eine Rolle spielt ${ }^{41}$.

Die Formen des Zusammenlebens von Einheimischen und Zuwanderern entziehen sich also politischen Regulierungen. Lebensweltliche Integration erfolgt oder sie erfolgt nicht. Wie aber kann man ihren Grad überhaupt messen? Wie kommt man zu einer einigermaßen zuverlässigen Datierung dieser Integrationsprozesse?

Hierzu gibt es verschiedene Indikatoren. Einer der aussagekräftigsten ist sicherlich das Heiratsverhalten, das in der Regel mehrere Phasen durchläuft. Auf die Berliner Hugenotten bezogene Untersuchungen ergeben hier ein klares Bild: Zunächst entstammten die Ehepartner der regionalen Landsmannschaft oder sogar dem gemeinsamen Herkunftsort der Refugiés ${ }^{42}$, sodann dem Kreis der Französischen Kolonie und schließlich der deutschen Bevölkerung, wobei im übrigen weitaus mehr französische Männer deutsche Frauen heirateten als deutsche Männer französische Frauen ${ }^{43}$. In Berlin kam es seit etwa $1760 \mathrm{zu}$ einer kontinuierlichen Zunahme von französisch-deutschen Mischehen ${ }^{44}$, was als ein Indiz für eine fortgeschrittene soziale Integration der dritten im Refuge geborenen Generation der Hugenotten gewertet werden kann. Ein weiterer Indikator zur Bemessung des Integrationsgrades der Hugenotten

${ }^{40}$ Unter anderem durch schulische und berufliche Förderprogramme sowie durch ein gesetzliches Diskriminationsverbot der Franzosen nichtfranzösischer Abstammung.

${ }^{41}$ So verlief zum Beispiel die soziale Integration der seit den 1950er Jahren nach Deutschland eingewanderten (christlichen) Italiener, Spanier, Portugiesen und Griechen weitgehend problemlos, während die Integration der (muslimischen) Türken und Kurden noch immer Schwierigkeiten bereitet; vermutlich hat dieser Umstand weniger mit der Integrationswilligkeit der betreffenden Gruppen als mit der Aufnahmebereitschaft der Mehrheitsgesellschaft zu tun.

${ }^{42}$ Vgl. Jürgen WILKE, Die Französische Kolonie in Berlin, in: Helga ScHULTZ, Berlin 16501800. Sozialgeschichte einer Residenz. Mit einem Beitrag von Jürgen Wilke [mit einer separaten Beilage: Tabellen], Berlin 1987, S. 352-430, hier S. 368f.

${ }^{43} \mathrm{Vgl}$. Eckart BIRNSTIEL, Andreas REINKE, Hugenotten in Berlin, in: JERSCH-WENZEL, JOHN, Von Zuwanderern zu Einheimischen (wie Anm. 9), S. 94-97. Dieses geschlechtsspezifische Heiratsverhalten ist auch in anderen Immigrantengruppen zu beobachten; möglicherweise handelt es sich hier um einen weiteren Integrationsschritt, dessen Qualität unbedingt einmal von Historikern, Soziologen und Anthropologen gemeinsam untersucht werden sollte: Ist die lebensweltliche Integration einer Gruppe erst dann vollzogen, wenn die ethnischen Proportionen in Mischehen ausgeglichen sind? Und handelt es sich dann überhaupt noch um »Mischehen«?

${ }^{44} \mathrm{Vgl}$. ibid., S. 96. 
ist sicherlich auch der Verlust der französischen Sprache, der sich in Preußen bezeichnenderweise ebenfalls ab der dritten Generation ankündigte ${ }^{45}$.

Den zuverlässigsten Indikator liefert aber offensichtlich die Remigrationsquote. Nur diejenigen Auswanderer kehren definitiv in ihr Herkunftsland zurück, deren persönliche Integration in der Fremde fehlgeschlagen ist ${ }^{46}$. In Hinblick auf die in Preußen niedergelassenen Hugenotten fällt in diesem Zusammenhang auf, daß deren Remigration schon in den 1760er Jahren praktisch völlig zum Erliegen $\mathrm{kam}^{47}$, und $\mathrm{daB}$ auch danach weder das Toleranzedikt Ludwigs XVI. noch das Reparationsgesetz der Französischen Nationalversammlung eine nennenswerte Rückwanderung der Hugenotten nach Frankreich auslösten. Daraus kann nur geschlossen werden, daß ihre Integration in die preußische Gesellschaft bereits seit den 1760er Jahren - also in der dritten Generation der Refugiés-Nachkommen - glücklich vollzogen war. Die »Krise der hugenottischen Identität« wurde also nicht erst durch die politischen Ereignisse der Französischen Revolution und der napoleonischen Besatzung Deutschlands provoziert ${ }^{48}$. Sie hatte wesentlich ältere Wurzeln.

\section{Nachbemerkung}

Jeder Vergleich historischer und aktueller Prozesse läuft Gefahr, sich in der Falle des Anachronismus zu verfangen. Die eingangs gestellte Frage - »Kann die Politik für die Integration ausländischer Mitbürger sorgen? « - entgeht dieser Gefahr nicht, denn das politische Instrumentarium monarchischer Obrigkeiten unterscheidet sich grundsätzlich von demjenigen demokratisch legitimierter Regierungen, und ständisch-feudal organisierte Gesellschaften funktionieren nach anderen Regeln als egalitär verfaßte.

Wenn dieser Vergleich jedoch den Sinn dafür geschärft hat, was Integration bedeutet und wie sie sich vollzieht, dann hat er seine Berechtigung. Er läßt jenseits aller historischen Befangenheiten ein Grundmuster erkennen: Wenn

${ }^{45}$ Vgl. Frédéric HARTWEG, Sprache, Identität, Nation. Das Refuge, Frankreich und Deutschland, in: BOHM, HĀSELER, VIOLET, Hugenotten zwischen Migration und Integration (wie Anm. 18), S. 155-166.

${ }^{46}$ In den heute in Deutschland und anderswo bestehenden Einwandererkolonien kann Integration selbstverständlich auch bedeuten, sich in parallelen Lebenswelten häuslich eingerichtet zu haben.

${ }^{47} \mathrm{Vgl}$. Eckart BIRNSTIEL, Le retour des huguenots du refuge en France, de la Révocation à la Révolution, in: Les protestants et la Révolution française (Sondernummer BSHPF [wie Anm. 16] 135 [1989]), S. 774-778.

${ }^{48}$ In diesem Sinne äußert sich Étienne FrançoIs, Vom preußischen Patrioten zum besten Deutschen, in: ThadDEN, Magdelaine, Die Hugenotten (wie Anm. 8), S. $198 \mathrm{f}$. 
die einheimische Bevölkerungsmehrheit eine Minderheit von Zuwanderern ablehnt, kann keine Politik der Welt deren glückliche Integration herbeiführen, so wie sie auch deren Integration durch nichts in der Welt verhindern kann, wenn diese auf die positive Reaktion der Mehrheit trifft.

Daraus ergibt sich, daß eine Politik der Integration von unbeliebten Minderheiten nur dann ihr Ziel erreicht, wenn sie sich auf die Mehrheit konzentriert. Wer die Integration »ausländischer Mitbürger« herbeiführen will, der muß die Aufnahmebereitschaft der einheimischen Bürger fördern. Beschränkt sich »Integrationspolitik « darauf, die der Minderheit durch ihre Ablehnung durch die Mehrheit entstehenden Nachteile auszugleichen, dann leistet sie lediglich dem Entstehen paralleler Lebenswelten Vorschub.

\section{Résumé français}

La question centrale à laquelle cet article se propose de fournir quelques éléments de réflexion est la suivante: quels étaient les mécanismes d'intégration des réfugiés huguenots ayant trouvé, dès la fin du XVIr siècle, asile en Brandebourg-Prusse? Leur intégration politique a-t-elle été voulue de la part du gouvernement? Dans quelle mesure l'image que la population allemande se faisait de ces immigrés français a-t-elle facilité leur intégration sociale? Comment peut-on mesurer leur degré d'intégration et quelle était la durée de leur acculturation, puis de leur assimilation à la population de leur pays d'accueil? Enfin, les mécanismes de l'intégration politique, sociale et culturelle des réfugiés huguenots peuvent-ils servir de modèle pour mieux comprendre ceux d'autres groupes d'immigrés, dans l'histoire comme dans l'actualité?

L'article veut aussi attirer l'attention sur quelques aspects de l'histoire du refuge huguenot qui, à l'heure actuelle, n'ont toujours pas été suffisamment pris en considération par la recherche: pourquoi la naturalisation des réfugiés huguenots n'a-t-elle été effectuée, dans les pays du refuge, qu'une génération après leur immigration massive? L'accroissement des mariages mixtes entre immigrés et autochtones, ainsi que le taux de rémigration en France des descendants des réfugiés huguenots, peuvent-ils servir de repères pour mesurer leur intégration? 